\title{
Isotropic Liquid Crystal Elastomers as Exceptional Photoelastic Strain Sensors
}

Devesh Mistry,* Maryam Nikkhou, Thomas Raistrick, Mariam Hussain, Ethan I. L. Jull, Daniel L. Baker, and Helen F. Gleeson

Cite This: Macromolecules 2020, 53, 3709-3718

Read Online

ACCESS I

Llll Metrics \& More

Article Recommendations

Supporting Information

ABSTRACT: A family of acrylate-based isotropic Liquid Crystal Elastomers (LCEs) exhibit stress- and strain-optic coefficients orders of magnitude greater than conventional polymeric and photoelastic materials. The three materials, composed of liquid crystalline and nonliquid crystalline monomers, show no nematic phase at any temperature. One of the materials has previously been synthesized with nematic symmetry, but here is instead templated with isotropic symmetry, demonstrating a previously unrealized idea proposed by de Gennes in 1969. Uniaxial strains applied to each material induce nematic ordering which we

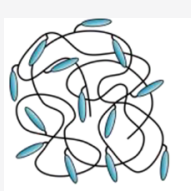

$\varepsilon \&<\mathrm{P} 2>=0$

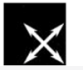

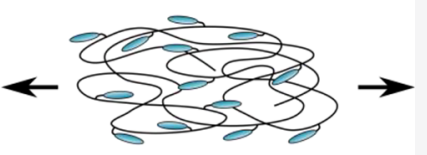

Increasing $\varepsilon \&<\mathrm{P} 2>\longrightarrow$

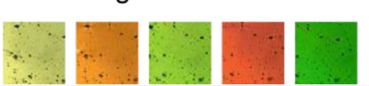
quantify using dye-absorption spectra and polarized Raman Spectroscopy. We deduce the coupling constants between the nematic liquid crystal order parameter and applied strain varies between $0.37 \pm 0.02$ and $0.66 \pm 0.02$ - values large compared to other LCE systems. The combination of high strain-optic coefficients $(0.048 \pm 0.003$ to 0.11 $\pm 0.01)$ and high compliances $\left(245 \pm 18\right.$ to $\left.1900 \pm 100 \mathrm{GPa}^{-1}\right)$ demonstrates that isotropic LCEs are exciting candidates for photoelastic coatings for assessing deformations across soft devices and biomaterials.

\section{INTRODUCTION}

Liquid crystal elastomers (LCEs) are remarkably responsive and biosimilar materials. Their inherent anisotropy leads to a variety of unique properties, including switchable geometries and soft elasticity. These outstanding features of LCEs have resulted in many exciting proposed applications, including photoactuated microrobots, novel chemical sensors, substrates for flexible electronics, and variable irises to name but a few. ${ }^{1-4}$ Indeed recently, some LCEs were found to exhibit negative Poisson's ratio, making them the first synthetic molecular auxetics. $^{5}$ Despite their wide range of possible applications, LCEs have rarely been considered as optical strain sensors. However, their exceptional combination of physical attributes suggests that, in principle, they could be ideal materials for studying and testing a wide variety of systems, including biological tissues and biomedical devices.

For such applications one cannot use conventional devices such as optical strain sensors as their size impacts the achievable spatial resolution. ${ }^{6-8}$ Consequently, alternative techniques are required for analysis of spatial stress distributions across materials and devices. ' While Finite Element Analysis (FEA) is often successfully used to simulate material deformations under loading, precise knowledge of material properties and structure are needed to faithfully model the system under study. ${ }^{10}$ By comparison, photoelasticity, which can map stresses and strains via the photoelastic effect, offers instantaneous and direct measurement of stress and strain distributions across complex physical objects and devices. ${ }^{11-13}$ Examples include the use of transmission photoelasticity to determine how stresses percolates through masonry walls modeled by transparent poly(methyl methacrylate) bricks and reflection photoelasticity to study the deformations of masonry columns and even dental prosthetics in vivo. ${ }^{14-16}$

Reflection photoelasticity offers huge potential in the field of biomedical engineering as it could be used to accurately determine the strain distributions across the surfaces of biological tissues and biomedical implant devices, and to create surrogate materials for studying biological tissues. Examples of previous studies to have used photoelastic methods to study soft biological tissues include Hirokawa et al. and Kawada et al., who studied strain in ligaments. ${ }^{17,18}$ Additionally, Fakhouri et al., and Doyle et al. used photoelastic experiments in conjunction with FEA analysis to respectively study the gap between the L4 and L5 vertebrae and models of abdominal aortic aneurysms., ${ }^{9,19}$ Lastly, Tomlinson et al. demonstrated using photoelastic materials as surrogate biological tissues in the study of tissue biomechanics. ${ }^{11}$ Going forward, photoelastic techniques could aid in the regulatory approval of biomedical devices, comparing the behaviors of natural and synthetic biomimetic materials in quasi-static and

Received: November 22, 2019

Revised: April 17, 2020

Published: May 6, 2020

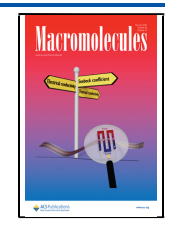


Table 1. Chemical Composition of Each LCE Precursor in Mol \%

\begin{tabular}{|c|c|c|c|c|c|c|c|c|}
\hline & \multicolumn{8}{|c|}{ component (mol \%) } \\
\hline & A6OCB & $6 \mathrm{OCB}$ & HDDA & M1 & M2 & RM82 & EHA & MBF \\
\hline LCE 1 & 14.6 & 55.9 & & & & 7.1 & 20.9 & 1.5 \\
\hline LCE 2 & 27.4 & 35.3 & 10.2 & & & & 25.6 & 1.5 \\
\hline LCE 3 & & & & 9.6 & 29.0 & 10.1 & 50.3 & 1.0 \\
\hline
\end{tabular}

\section{Monofunctional mesogenic acrylates}<smiles>C=CC(=O)OCCCCCOc1ccc(-c2ccc(C#N)cc2)cc1</smiles><smiles>[M]CCCCOC(=O)C=C</smiles>

M2<smiles>C=CC(=O)OCCCCCOc1ccc(OC(=O)c2ccc(OC)cc2)cc1</smiles>

Non-mesogenic monofunctional acrylate

EHA<smiles>C=CC(=O)OCC(CC)CCCC</smiles>

Bifunctional crosslinkers

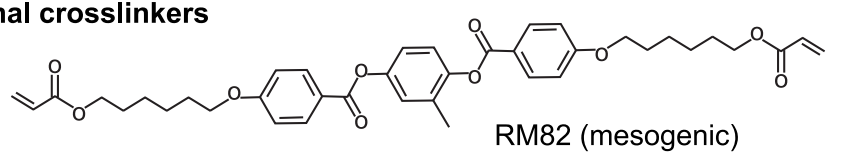

\section{Other}

$60 \mathrm{CB}$

(mesogenic solvent)

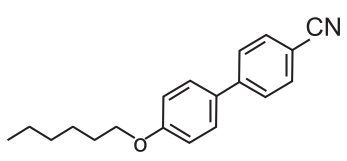

15 NB3

(dichroic dye) (photoinitiator)

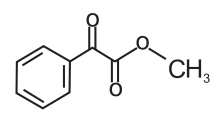

HDDA (non-mesogenic)

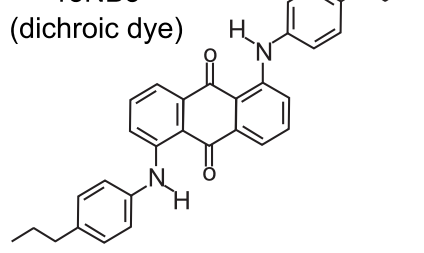

Figure 1. Chemical components used to create the LCEs.

dynamic testing and also in verifying the accuracy FEA models of biomedical devices that are fabricated from smart and multifunctional materials with complex mechanical behvaviours. $^{9,19,20}$

However, one of the major challenges for employing photoelastic methods is choosing materials with appropriate physical properties. For reflection photoelasticity, low modulus (high compliance) materials with large strain-optic coefficients are required. Such materials balance minimal stress-shielding effects and sufficiently large photoelastic responses for accurate localized strain measurements. ${ }^{21}$ Additionally, a chosen photoelastic material must be able to match the strains of the system under test. ${ }^{11,17}$ The unique properties of liquid crystal elastomers makes them exciting candidates for photoelastic strain measurement.

There have been a few reports of liquid crystal elastomers employed as optical strain sensors. Picot et al. described a realtime optical strain sensor that uses a relatively highly crosslinked chiral nematic LCE. ${ }^{22}$ In their work, a 5-7 $\mu$ m thick LCE film was spray-coated onto uniaxially oriented films of polyamide- 6 that provided both a substrate and the necessary alignment of the liquid crystalline structure. Applied strains caused a shift in the selective reflection wavelength associated with the chiral nematic helicoidal structure. Although no special illumination was required to detect the optical response of the LCE, the material's applications are limited by the need for an alignment layer to the orient the LCE.

In their early studies into polysiloxane- and polymethacrylate-based LCEs, the Finkelmann group observed that stresses applied to nematic LCEs just above their nematic-to-isotropic transition temperature, $T_{\mathrm{NI}},\left(\sim 11{ }^{\circ} \mathrm{C}\right.$ and $\sim 60{ }^{\circ} \mathrm{C}$ for the polysiloxane and polymethacrylate LCEs, respectively) resulted in a large photoelastic response. ${ }^{23,24}$ The authors determined that stresses applied to these LCEs in their respective isotropic phases caused a softening of the isotropic transition and the establishment of a "paranematic" phase. ${ }^{23-25}$ While the Finkelmann materials show that LCEs could form the basis of a photoelastic sensor, their materials' photoelastic response were highly temperature dependent. In addition, the synthetic route of the polysiloxane materials makes them difficult to use as coatings and the high $T_{\mathrm{NI}}$ of the polymethacrylate materials severely limits their application.

Recently, there has been a growing interest in isotropic elastomeric networks containing liquid crystal moieties. ${ }^{26-28}$ Donovan et al. described strain-induced nematic phases in thiol-ene LCEs which demonstrated a nematic order parameter, $\langle P 2\rangle$, of $\sim 0.3$ at the material's maximum strain of 2.25, a value most likely indicative of a biphasic state containing paranematic and nematic phases. ${ }^{29}$ Their study found that their isotropic LCEs had a significantly greater toughness, less creep, and faster recovery times compared to polydomain LCEs of similar chemistry-although comparisons with the performance of conventional isotropic elastomers were not made. ${ }^{26}$

In this work, we describe three isotropic LCE-based materials that demonstrate both exceptionally large photoelastic responses and high compliances when compared to conventional polymers and elastomers, and commercially available photoelastic coatings. The materials are related to a recently reported nematic acrylate-LCE, but here they are templated with isotropic symmetry and so show no liquid crystalline behavior as a function of temperature. ${ }^{27,30}$ As our materials are synthesized via photopolymerization of commercially available acrylate monomers, they are scalable and would be readily used as photoelastic coatings. Compared to other LCE optical sensors, with our materials there is no need for 
Table 2. Approximate Mol \% Composition of Each Final LCE Following Washing of Unreacted Components (MBF Neglected)

\begin{tabular}{|c|c|c|c|c|c|c|c|}
\hline & \multicolumn{6}{|c|}{ component (mol \%) } & \multirow[b]{2}{*}{ mesogenic fraction $(\mathrm{mol} \%)$} \\
\hline & $\mathrm{A} 6 \mathrm{OCB}$ & HDDA & M1 & M2 & RM82 & EHA & \\
\hline LCE 1 & 34 & & & & 17 & 49 & 51 \\
\hline LCE 2 & 43 & 16 & & & & 41 & 43 \\
\hline LCE 3 & & & 10 & 29 & 10 & 51 & 49 \\
\hline
\end{tabular}

alignment layers to control liquid crystal orientation as the monomer precursor mixture and unstrained final materials have isotropic symmetry. Additionally, the photoelastic response of our materials has minimal temperature dependence near ambient temperatures. Lastly, when mechanically strained, our materials display a classical isotropic rubber response as opposed to the soft elastic behavior common to polydomain nematic rubbers. ${ }^{31-33}$ We propose that these unique combination of properties makes the materials described particularly suited to the study of the strain distributions across soft biological tissues and biomedical devices.

\section{EXPERIMENTAL METHODS}

Materials Synthesis. Isotropic LCEs were synthesized using various compositions of the following components-shown in Table 1. 6-(4-Cyano-biphenyl-4'-yloxy)hexyl acrylate (A6OCB), 4-(6acryloyloxy)hexyloxy)phenyl 4-(trans-4-propylcyclohexyl)benzoate (M1), and 4-methoxybenzoic acid 4-(6-acryloyloxyhexyloxy)phenyl (M2) are monofunctional mesogenic acrylates. The mesogenic structures of these molecules means they have high anisotropy in their optical polarizabilities that in turn contribute to a high birefringence when orientational alignment is present. As applied strains align these molecules, these mesogenic groups are expected to drive large photo elastic responses. 2-Ethylhexyl acrylate (EHA) is a monofunctional acrylate, a polymer of which has a low glass transition temperature $\left(T_{\mathrm{g}}\right)$ of approximately $-65{ }^{\circ} \mathrm{C} .{ }^{34}$ As acrylate LCEs are typically brittle at ambient temperatures, EHA serves as a plasticizing group allowing low- $T_{\mathrm{g}}$ materials to be synthesized. ${ }^{30}$ 1,4-Bis-[4-(6acryloyloxyhexyloxy) benzoyloxy]-2-methylbenzene (RM82) and 1,6hexanediol diacrylate (HDDA) are bifunctional acrylate cross-linking groups which join polymer chains to form elastomeric materials. RM82 has the added advantage of having a mesogenic core which can also contribute to the photoelastic response of the relevant materials. 4-Cyano-4' - hexyloxybiphenyl (6OCB) is a nonreactive nematic compound and is included in LCEs 1 and 2 to prevent phase separation. Methyl benzoylformate (MBF) is a UV-reactive photoinitiator and $15 \mathrm{NB} 3$ is an anthraquinone dichroic dye used in small concentrations for measuring the $\langle\mathrm{P} 2\rangle$ order parameter via the guesthost effect as described below. Figure 1 shows the chemical structure of each compound used in this work. A6OCB, M1, M2, and RM82 were purchased from Synthon Chemical GmbH while HDDA, EHA, and MBF were purchased from Sigma-Aldrich. The 15NB3 dichroic dye was provided by the School of Chemistry at York University. ${ }^{35}$ All chemicals were used without further purification. Table 2 shows the approximate monomer compositions for LCEs 1-3 following the removal of 6OCB from LCEs 1 and 2.

LCE 1 has the same chemistry as the material studied in refs: 30,5 , and 36. In this work, we polymerized the material at $50{ }^{\circ} \mathrm{C}$-in the isotropic phase of the monomer mixture, $13.4{ }^{\circ} \mathrm{C}$ above the nematic to isotropic phase transition temperature, $T_{\mathrm{N}}$, deduced by Differential Scanning Calorimetry, (described below, data are provided in Figure S1 of the Supporting Information, SI). We prepared LCE 2 and LCE 3 using monomer compositions and a method like that used for LCE 1. The LCE precursors were polymerized inside thin film molds of $\sim 100 \mu \mathrm{m}$ thickness and were removed from the molds following polymerization. Additionally, LCE 1 and LCE 2 (swollen with 6OCB) were washed of their $6 \mathrm{OCB}$ content as previously described. ${ }^{30}$ LCE 3 therefore has the advantage of being ready for use after polymerization with no washing stage required.

Differential Scanning Calorimetry. The glass transition temperatures of the polymers were determined via Differential Scanning Calorimetry (DSC) using a TA Instruments Q20 DSC. Samples were heated at rate of $10^{\circ} \mathrm{C} \mathrm{min}^{-1}$ between -40 and $60{ }^{\circ} \mathrm{C}$. The first heating run erased the thermal history of the tested samples. $T_{\mathrm{g}}$ was taken from the inflection point of the baseline shift from the second heating run. DSC also serves to investigate whether the elastomers exhibit any liquid crystal phases as nematic (or any other LC) to isotropic phase transition temperatures would be clear as endothermic (exothermic) peaks on heating (cooling). The nematic to isotropic transition temperature, $T_{\mathrm{N}}$, of the monomer precursor to LCE 1 was confirmed by scanning a sample of the uncured resin between 0 and $50{ }^{\circ} \mathrm{C}$.

Cyclical Mechanical Testing. To study the repeatibility of the LCEs' photoelastic responses we performed cyclical mechanical testing measuring the color of the samples and the peak stresses at half of the measured failure strain of each LCE $(42 \%, 32 \%$, and $18 \%$, respectively, see Figure S2). Samples of high aspect ratio (length/ width $>10$ ) were loaded into an Instron 5560 mechanical tester. Samples were cycled until failure (up to a maximum of 100 cycles) at a strain rate of $1 \% \mathrm{~s}^{-1}$.

Photoelastic Characterizaton. We quantified the photoelastic response of the elastomers and the stress-induced nematic order using bespoke apparatus manufactured in-house and described in ref 30 and its associated data set. The apparatus allows simultaneous observation of the sample via polarizing microscopy during mechanical testing. A linear (uniaxial) strain is employed in these experiments on test samples prepared with a high aspect ratio (length/width $>10$ ). Each test was performed quasi-statically as after each strain step, the sample was allowed to stress relax for $2 \mathrm{~min}$ before measurements were performed. We captured transmitted white light and polarizing microscopy photographs (with the crossed polarizers placed at $45^{\circ}$ to the imposed stress axis) at each strain step. We measured localized strains parallel and perpendicular to the deformation axis from the white light images using ImageJ by determining the fractional change in separation between particles (micron-scale artifacts formed during synthesis) embedded within the LCE films. Errors on the localized strains were calculated from the positional error of the tracer particles used to measure localized strain, which was typically a 2 or 3 pixel error on each of the particle positions. For each sample at each strain step, we used a Berek compensator to measure the stress-induced optical retardance. Dividing the retardance value by the sample thickness at a given strain step yielded the sample birefringence. The film thickness with strain was determined under an assumption of constant volume by dividing the initial sample thickness (measured using a calibrated micrometre with $1 \mu \mathrm{m}$ accuracy) by the product of the deformations parallel $\left(\lambda_{x}\right)$ and perpendicular $\left(\lambda_{y}\right)$ to the imposed stress axis. The shear-free constant volume assumption has been tested thoroughly for this family of acrylate LCEs, confirming that this approach is robust. ${ }^{5,30,36}$

Order Parameter from Dichroic Absorption. The dichroic absorption experiments require a dye-doped sample, so $0.1 \mathrm{wt} \%$ anthraquinone dichroic dye (15NB3) was added during the synthesis of a sample of LCE 2 to measure the order parameter $\langle P 2\rangle{ }^{37}$ The incorporation of the dye into the material results in the guest-host effect, with the dye molecules aligning with the host. ${ }^{38,39}$ At each strain step, the transmission spectra were measured with a single polarizer parallel or perpendicular to the strain axis. The absorption values at $0^{\circ}\left(A_{\|}\right)$and at $90^{\circ}\left(A_{\perp}\right)[\lambda=550 \mathrm{~nm}]$ were then used to 
calculate the order parameter of the bulk material. ${ }^{40}$ We performed two experiments on LCE 2 with the testing chamber temperature maintained 22 and $10{ }^{\circ} \mathrm{C}$.

Order Parameter from Polarized Raman Spectroscopy. Polarized Raman Spectroscopy (PRS) was also used to determine the order parameter of each LCE with strain. The relevant order parameter is $\langle P 2\rangle=\left\langle\frac{1}{2}\left(3 \cos ^{2} \theta-1\right)\right\rangle$ (the angular brackets imply an ensemble average and $\theta$ is the angle of the molecules with respect to the director). $\langle P 2\rangle$ can be obtained from measurements of the depolarization ratio in PRS; in this case the $1606 \mathrm{~cm}^{-1}$ Raman line was used for analysis. ${ }^{41-43}$ The Raman depolarization data were obtained using a polarized Raman system (Renishaw InVia) with a $532 \mathrm{~nm}, 50 \mathrm{~mW}$ solid state laser. The sample was stretched using a manual stretching rig, mounted on the rotating stage of the microscope (Leica DM2700), and was illuminated in reflection by a $20 \times$ objective lens. Five $\mathrm{mW}$ of laser power was incident at the sample, and a $10 \mathrm{~s}$ exposure time was used to achieve an optimal signal/noise ratio. The data were analyzed as described in refs 43 and 44 using the full depolarization ratio to deduce $\langle P 2\rangle$.

\section{THEORY}

Classical Isotropic Elasticity. For conventional isotropic elastomers, one expects the stress-strain response to behave as follows:

$$
\sigma^{\mathrm{e}}=\mu\left(\lambda-\frac{1}{\lambda^{2}}\right)
$$

where $\sigma^{\mathrm{e}}$ is the engineering stress, $\mu$ is the elastic modulus, and $\lambda$ is the applied deformation which is equivalent to $\epsilon+1$ where $\epsilon$ is the applied strain. The Young's modulus, $E$, is found from the derivative of eq 1 at zero strain $(\lambda=1)$,

$$
E=3 \mu
$$

Assuming the elastic body deforms at constant volume and with minimal shears, the elastic modulus, $\sigma^{e}$ can be linked to the true stress, $\sigma^{t}$ (stress based on the current cross sectional area as opposed to the initial cross sectional area), via:

$$
\sigma^{\mathrm{t}}=\lambda \times \sigma^{\mathrm{e}}=\mu\left(\lambda^{2}-\frac{1}{\lambda}\right)
$$

Neo-classical Theory of LCEs. The Warner and Terentjev neoclassical theory of nematic LCEs offers a simple yet robust way to study the mechanical properties of nematic LCEs and extract physical observables to compare against experiment. ${ }^{5,44,45}$ Here, we apply the Warner and Terentjev theory to develop an understanding of the evolution of the straindependent order parameter.

In their theory, the (average) anisotropic nature of an LCE's polymer conformation is captured by modeling the polymer backbone using an anisotropic random walk. For example, in typical nematic materials with positive order parameter, the polymer chain takes larger steps in the direction parallel to the director and so the random walk step length is larger in this direction than in directions perpendicular to the director. The anisotropy of the random walk is encapsulated in the step length tensor, $\underline{\underline{l}}$. Under this framework, one finds the free energy of a LCE is given by the trace formula: ${ }^{44}$

$$
F=\frac{1}{2} \mu \operatorname{Tr}\left(\underline{\underline{l}}_{0} \cdot \underline{\underline{\lambda}}_{t}^{T} \cdot \underline{\underline{l}}^{-1} \cdot \underline{\underline{\lambda}}_{t}\right)
$$

where $\mu$ is the elastic modulus, $\underline{\underline{l}}_{0}$ and $\underline{\underline{l}}$ are, respectively, the step length tensors in the unstrained and strained states and $\underline{\underline{\lambda}}_{t}$ is the deformation gradient tensor which for shear-free deformations is diagonal and has principal elements, $\lambda_{i}=\epsilon_{i}$ +1 where $\epsilon_{i}$ is the relevant principal strain.

In this work, our unstrained materials are isotropic, hence $\underline{\underline{l}}_{0}=\underline{\underline{I}}$-the identity matrix. If a shear-free and volume conserving deformation is applied along the $x$ axis, then,

$$
\begin{aligned}
& \underline{\underline{\lambda}}_{t}=\operatorname{diag}\left(\lambda_{x}, \lambda_{y}, \frac{1}{\lambda_{x} \lambda_{y}}\right), \text { and } \\
& \underline{\underline{l}}=\operatorname{Diag}\left(l_{\|}, l_{\perp}, l_{\perp}\right)
\end{aligned}
$$

where the symmetry of the system means the unique axis of the step length tensor lies parallel to the deformation axis and the step lengths $l_{y}$ and $l_{z}$ are equal. Additionally, in eq 5, we have used the shear-free condition of volume conservation $\lambda_{x} \lambda_{y} \lambda_{z}=$ 1 to eliminate $\lambda_{z}$.

Minimizing the free energy of the trace formula with respect to the deformations $\lambda_{y}$ and $\lambda_{x}$ one finds,

$$
r=l_{\|} / l_{\perp}=\lambda_{x}^{3}=\left(\epsilon_{x}+1\right)^{3}
$$

where $r$ is the step length anisotropy-a measure of the anisotropy of a LCE that is readily measured from experimental data.

Finkelmann et al. showed that one can create an order parameter for the polymer backbone, $Q_{B}$, which is linked to the step length anisotropy via, ${ }^{46}$

$$
Q_{B}=\frac{r-1}{r+2}
$$

Inserting eq 7 yields the following prediction linking $Q_{\mathrm{B}}$ to the applied strain,

$$
Q_{\mathrm{B}}=\frac{\left(\epsilon_{x}+1\right)^{3}-1}{\left(\epsilon_{x}+1\right)^{3}+2} \approx \epsilon_{x}
$$

where the approximation is valid for low strains of $\epsilon_{x} \lesssim 0.4$ (see Figure S3). For side-chain LCE systems such as those studied here, the nematic order parameter, $Q_{N}$, is proportional to the polymer bone order parameter, $Q_{B} \cdot{ }^{46}$ Hence, for low strains one should expect the following:

$$
Q_{\mathrm{N}}=\alpha \cdot \epsilon_{x}
$$

Photoelasticity. It is well-known that a stress applied to an isotropic polymeric material induces a small degree of material anisotropy; for a transparent material this can be evident through the emergence of birefringence. ${ }^{47-49}$ For isotropic materials, it is expected that the induced birefringence, $\Delta n$, will be proportional to the true stress, $\sigma^{\mathrm{t}}$, with the constant of proportionality defined as the stress-optical coefficient, $C{ }^{23,50}$

$$
\Delta n=C \sigma^{\mathrm{t}}
$$

A similar relationship exists linking the induced birefringence to the differences in principle strains in the observation plane, with the constant of proportionality defined as the strain-optic coefficient, $K_{;}^{51}$

$$
\Delta n=K \Delta \epsilon
$$

\section{RESULTS}

Glass Transitions of the Polymers and Thermal Phase Transitions of the Monomer Mixtures. Figure 2a shows DSC traces for the three isotropic LCEs. None of the materials exhibit endothermic/exothermic peaks that would indicate an 


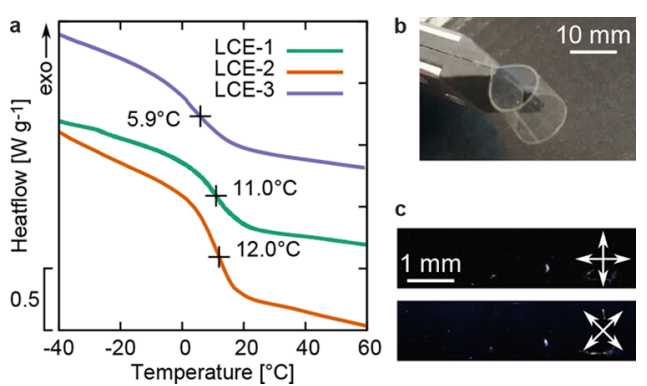

Figure 2. (a) DSC traces for LCEs 1, 2, and 3 with their respective glass transitions marked. (b) Photograph of a film of LCE 3 demonstrating both flexibility and transparency. The film is approximately $10 \mathrm{~mm}$ wide and $100 \mu \mathrm{m}$ thick. (c) Example polarizing optical microscopy images of LCE 3. LCEs 1 and 2 are also isotropic and appear black when viewed via polarizing optical microscopy. These photographs confirm the transparency of the material as scattering centers from impurities would be clearly visible in these photographs.

isotropic-nematic phase transition. The glass transition temperatures of the materials are $11.0 \pm 1.0{ }^{\circ} \mathrm{C}, 12.0 \pm 1.0$ ${ }^{\circ} \mathrm{C}$, and $5.9 \pm 1.0^{\circ} \mathrm{C}$ for LCEs 1,2 , and 3 respectively. We also examined the phase behaviors of each LCE precursor optically and via DSC scans performed between 0 and $50{ }^{\circ} \mathrm{C}$. The precursor of LCE 1 appeared (in bulk) as a typical lightscattering liquid crystal. From DSC we measured the nematic to isotropic phase transition at $36.6 \pm 1.0{ }^{\circ} \mathrm{C}$ (Figure S1). ${ }^{30}$ The precursors of LCE 2 and LCE 3 show no liquid crystalline behavior as they were optically isotropic liquids and showed no liquid crystal phase transitions via DSC.

Optical Properties of the Polymers. All the polymer samples studied were optically transparent both with and without applied strain. Figure $2 b, c$ shows optical images of LCE 3 which is typical of all the polymers. Figure $2 b$ shows a representative image of a film of LCE 3 demonstrating the LCEs soft, flexible, and transparent nature. Figure $2 \mathrm{c}$ shows polarizing optical microscopy images of an unstrained sample of LCE 3. The material appears black as the polarizers are rotated by $45^{\circ}$ - confirming that material must be either optically isotropic or in a nematic phase with a homeotropic geometry. We can easily discount the latter option since no transition temperatures were seen via DSC and because when viewed via conoscopy, the material remains black with no "Maltese cross" pattern present. Similar observations were made for LCEs 1 and 2. Figure 2c also confirms the highly transparent nature of the LCE as any scattering centers of light (for example microscopic domains of nematic order or defect structures) would be clearly visible here, and would also make the sample appear white or translucent to the naked eye.

Figure 3 shows polarizing microscopy images of LCE 3 (note, this sample studied did not contain any of the $15 \mathrm{NB} 3$ dye) during mechanical testing, similar data for LCEs 1 and 2 are shown in Figure S4. Applied strains cause all the polymers to become birefringent with approximately two orders of birefringence colors readily observable in the $100 \mu \mathrm{m}$ thick films over the strains tested. For all three of the materials, the observed progression of birefringence colors indicates a continuous increase in the nematic ordering as a function of strain.

Quasi-static Mechanical and Photoelastic Behavior. Figure 4a shows the true stress-strain tensile load curves of the polymers. We plot true stress given its use in calculating

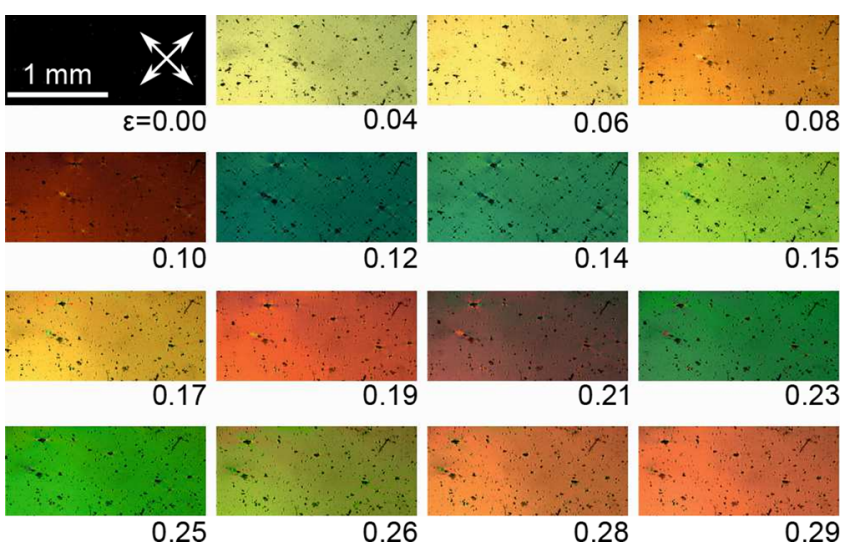

Figure 3. Strain-induced birefringence colors for LCE 3 as seen via crossed polarizers. The strain is applied in the horizontal direction for the sample as shown. A similar progression of strain-induced birefringence colors are seen with LCEs 1 and 2 (see SI Figure S4).

photoelastic constants. Using eq 2 and values of $\mu$ from fitting eq 3 to the true stress-strain data, we calculate that LCE 1 , LCE 2, and LCE 3 have Young's moduli of $4.1 \pm 0.3,0.53 \pm$ 0.03 , and $1.11 \pm 0.15 \mathrm{MPa}$ (compliances of $245 \pm 18,1900 \pm$ 100 , and $\left.900 \pm 120 \mathrm{GPa}^{-1}\right)$, respectively. Such values are comparable with soft rubbers and biological tissues such as cartilage $(E \approx 1 \mathrm{MPa}) .^{52}$

Figure $4 \mathrm{~b}$ shows the strain-induced birefringence of LCE 1, LCE 2, and LCE 3. All three materials are well-fitted by a linear curve with gradients of $0.15 \pm 0.02,0.068 \pm 0.005$, and 0.084 \pm 0.009 , respectively (note these are values are not the strainoptic coefficients). By using these values and approximating the true stress curves as linear curves, we deduce stress-optical coefficients for LCE 1, LCE 2, and LCE 3 of the following:

$$
\begin{aligned}
& C_{1}=(0.037 \pm 0.006) \times 10^{-6} \mathrm{~Pa}^{-1}=(3.7 \pm 0.6) \times 10^{4}, \quad \text { Brewsters } \\
& C_{2}=(0.13 \pm 0.01) \times 10^{-6} \mathrm{~Pa}^{-1}=(13 \pm 1) \times 10^{4}, \quad \text { Brewsters and } \\
& C_{3}=(0.075 \pm 0.013) \times 10^{-6} \mathrm{~Pa}^{-1}=(7.5 \pm 1.3) \times 10^{4}, \quad \text { Brewsters }
\end{aligned}
$$

respectively. For comparison, the stress optical coefficient of most polymeric materials is $\sim 10^{3}$ Brewsters; our polymers are one to 2 orders of magnitude higher.

For calculating the strain-optic coefficients of LCEs 1-3, we used the gradient of linear fits to the birefringence against the measured difference in principle strains (Figure S5). For LCEs 1,2 , and 3 , we determined strain-optic coefficients, $K$, of 0.11 $\pm 0.01,0.048 \pm 0.003$, and $0.06 \pm 0.01$, respectively.

Strain Dependent Order Parameter. Figure 4c shows the dependency of the nematic order parameter, $\langle\mathrm{P} 2\rangle$, on strain as measured via PRS. The behavior of each material is approximately linear thus from the gradients at low strain $(\epsilon<$ 0.4 ) we can extract values for the coupling term $a$ shown in eq 10. For LCEs 1,2 , and 3, we measure $a$ to be $0.51 \pm 0.02,0.37$ \pm 0.02 , and $0.66 \pm 0.02$, respectively. In addition, as LCE 3 does not require the presence of the nonreactive group $6 \mathrm{OCB}$ during synthesis, the material could be doped with the dye $15 \mathrm{NB} 3$ allowing us to measure, at different temperatures, the LCE order parameter via the guest-host effect (Figure 4d). For both tests performed at 10 and $22{ }^{\circ} \mathrm{C}$ the coupling between the order parameter and the mechanical field appears linear as predicted by eq 10 . Using linear fits, we obtain values of $a=0.71 \pm 0.02$ and $0.74 \pm 0.05$ for at 10 and $22{ }^{\circ} \mathrm{C}$, respectively, values which agree well with each other and the 

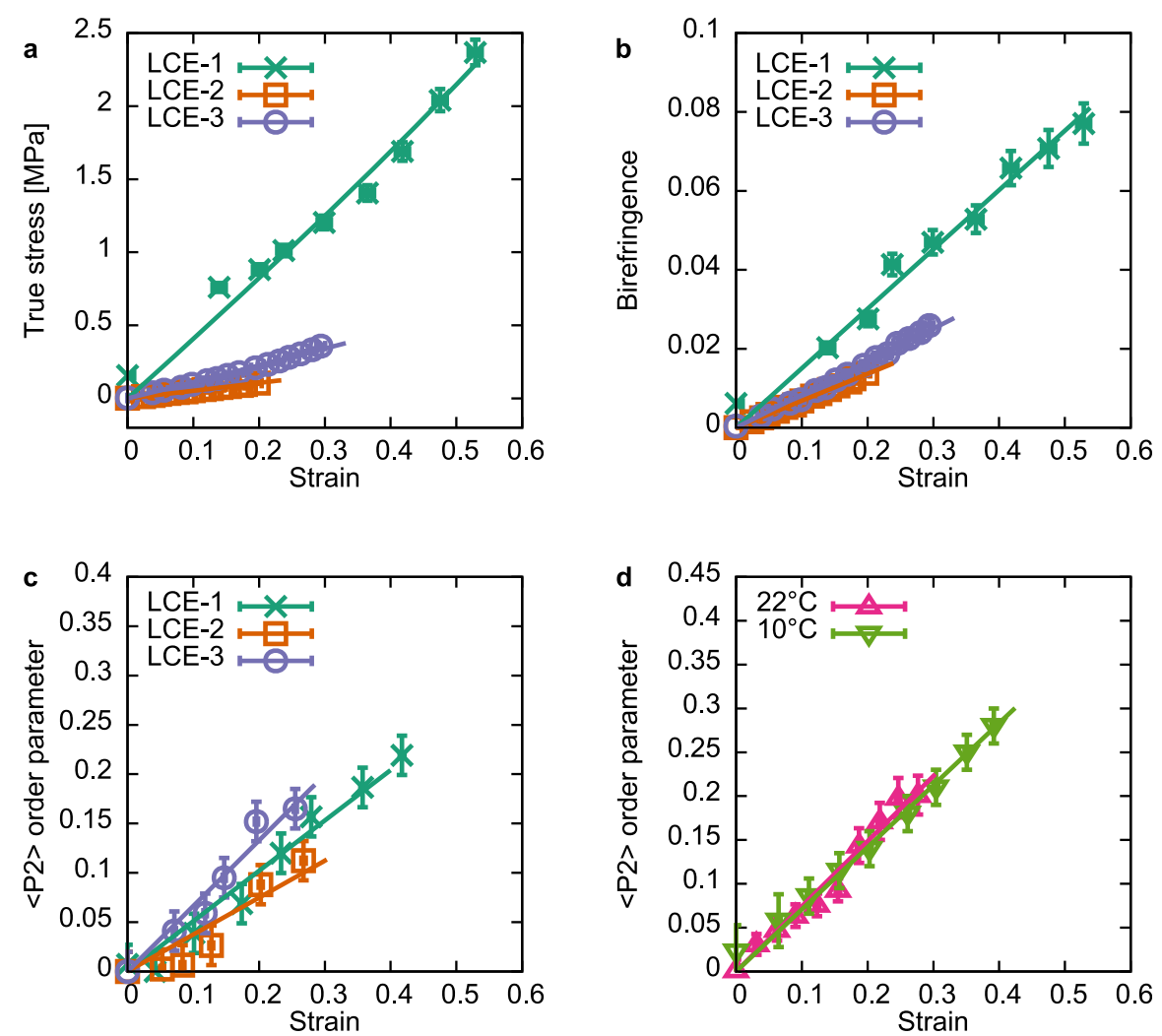

Figure 4. For LCEs 1, 2, and 3, (a) true stress-strain tensile data, (b) birefringence-strain data, and (c) the uniaxial order parameter, $\langle\mathrm{P} 2\rangle$, as a function of the strain as measured using PRS. (d) For LCE 3, order parameter deduced from absorption measurements in dye-doped LCE 2 at room temperature and $10{ }^{\circ} \mathrm{C}$.

value obtained via PRS. The agreement between these values of $a$ at 10 and $22{ }^{\circ} \mathrm{C}$ shows the proportionality constant $a$ and the strain dependent order parameter are constant at around ambient temperatures. Figure S6 shows for the test performed on LCE 3 at $10{ }^{\circ} \mathrm{C}$, visible light absorption spectra for the unstrained and maximally strained states. While straining causes the overall intensity of polarized light absorbed parallel (perpendicular) to increase (decrease) (Figure S6a), the overall wavelength profile (color) of light absorbed parallel and perpendicular to the strain axis remains equal (Figure S6b). The ratio of these absorptions can therefore be used to measure the order parameter at each strain step.

Cyclical Mechanical and Optical Consistency. Figure 5 demonstrates the consistency of the tensile mechanical

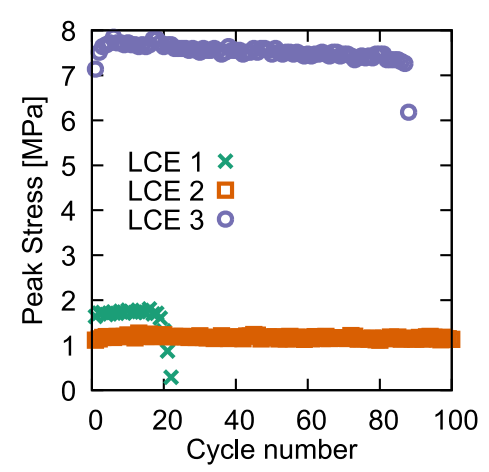

Figure 5. Consistency of peak stresses through cyclical testing for LCEs $1-3$ respectively. Each sample was cyclically strained to $50 \%$ of the measured failure strain (Figure S2). behaviors of LCEs-1-3 via cyclical testing, repectively. While LCE 1 failed after 20 cycles, LCE 3 lasted for 88 cycles, while LCE 2 lasted the full 100 cycles of testing without failure. For the first 20 cycles of testing, all three materials demonstrate consistent peak stresses of 1.8, 1.0, and 7.7 MPa for LCEs 1, 2, and 3 respectively, although LCE 2 shows evidence of strain hardening in the first few cycles. Past 20 cycles, the peak stress of LCE 2 remained at $1.0 \mathrm{MPa}$ while for LCE 3 the peak stress gradually decreased to about $7 \mathrm{MPa}$. SI Videos $\mathrm{S} 1-\mathrm{S} 3$ show, for LCEs 1, 2, and 3, respectively, photographs of the samples (between crossed polarizers) taken during the first four cycles of cyclical testing. As the color progressions with cyclical strain repeat themselves, these videos qualitatively show the repeatability of the photoelastic response of our materials.

\section{DISCUSSION}

In this study, three isotropic LCEs were synthesized, and their mechanical, photoelastic, and phase behaviors were compared. In all cases, the LCE precursors contain a mixture of mesogenic and nonmesogenic acrylate monomers and were all polymerized in the isotropic phase. LCE 1 is the same material previously studied-but templated with isotropic symmetry. LCE 2 is like LCE 1 but with a nonmesogenic crosslinker. LCE 3 uses different mesogenic monomers to LCEs 1 and 2 and has a composition that does not require the nematic solvent $6 \mathrm{OCB}$ to form a phase stable mixture for network formation. Our results demonstrate fundamental insights into the phase behavior of LCEs and potential for application as photoelastic stress sensors.

Photoelastic Applications. A key result of this study is that our LCEs demonstrate stress optical coefficients $\sim 100 \times$ 
greater than that typically observed in conventionally isotropic elastomers (Figure 6a). While the materials described by the
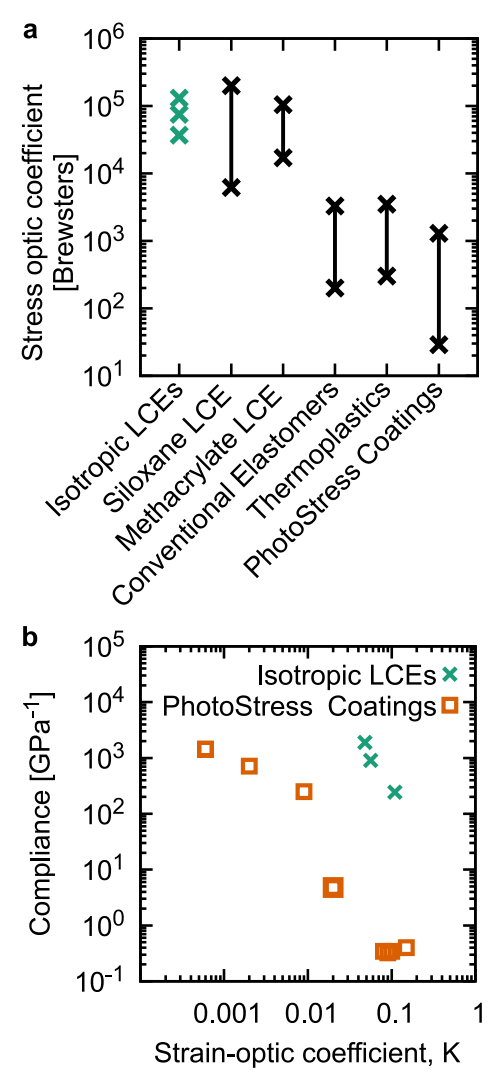

Figure 6. (a) Comparison of the stress optic coefficient of LCEs 1-3 with other LCEs and polymeric materials. The range of values shown for the Finkelmann group's siloxane and methacrylate LCEs reflects these materials' temperature sensitivity. The methacrylate LCE also has a glass transition temperature of $60{ }^{\circ} \mathrm{C}$. (b) Comparisons of the performance of our isotropic LCEs against commercially available PhotoStress photoelastic coatings. Ideal photoelastic coatings have high compliances (low moduli) and high stress-optic coefficients. For references and more information, see Tables S1 and S2.

Finkelmann group demonstrate similar stress-optic coefficients, they are not suitable for application as photoelastic coatings due to their complex synthesis routes, limitations of a nematic phase, and high temperature sensitivity of the stress-optic coefficient. ${ }^{23,24}$ Additionally, the polymethacrylate material had a high nematic-to-isotropic transition temperature $\left(60{ }^{\circ} \mathrm{C}\right)$, making it unsuitable for tests performed at ambient conditions, and has a nonlinear relationship between strain and induced birefringence (when held at a fixed temperature). ${ }^{24}$ We also note that our LCEs are all well fitted by eq 2 , which gives the expected tensile response for a classical isotropic elastomer. Our LCEs would therefore be simple to model and work with in photoelastic experiments. By comparison, conventional LCEs are well-known for their highly complex nonlinear and anisotropic mechanical behaviors. $3,26,36,44,45$

The PhotoStress materials from MicroMeasurements VPG are common materials currently used in photoelastic experiments. The range of materials available varies from high modulus (2.5 GPa) systems with high strain-optic coefficients, $\mathrm{K}(0.15)$ to low modulus materials $(0.7 \mathrm{MPa})$ with low values of $K\left(6 \times 10^{-4}\right)$. For photoelastic strain detection, optimal materials will have high strain optic coefficients for producing strong signals while having high compliances (inverse of modulus) to minimize the stress-shielding effects of the photoelastic coating. Figure $6 \mathrm{~b}$ uses an Ashby-type plot to compare the compliance and stress optic coefficients of LCEs 1-3 with the range of PhotoStress materials. Figure 6 clearly shows that the PhotoStress materials all fall on one line where one must choose between materials with a high compliance and high strain-optic coefficient. LCEs 1-3 appear to follow a similar trend, however their position on the Ashby plot is shifted by an order of magnitude greater along the compliance and strain-optic axes. Thus, the present materials belong to a completely different class of photoelastic material-clearly outperforming all the PhotoStress materials.

We can explain the differences in the strain-optic coefficients, $K$, of LCEs 1,2 , and 3 (0.11 $\pm 0.01,0.048 \pm$ 0.003 , and $0.06 \pm 0.01$, respectively) by considering the differences in the mesogenic composition of each LCE. LCE 1 has the greatest stress-optic coefficient as it has the highest mesogenic content (51 mol.\%, Table 2) and it uses A6OCB, a mesogen that contains the highly polar biphenyl group (Figure 1). This chemical group means LCE 1 has a high optical anisotropy when the mesogens are aligned. Despite LCE 3 having the strongest coupling, $a$, between the polymer backbone and mesogenic ordering, its photoelastic response is only marginally greater than that of LCE 2 . We attribute this to the fact that none of the mesogenic components have particularly high optical anisotropy. Considering the photoelastic response and backbone-nematic order parameter coupling together, one could envisage creating a photoelastic material with an even greater photoelastic response than LCE 1 by choosing similarly structured monomers as M1 and M2 but with highly polarizable groups.

Of our materials, LCE 3 would be the simplest material to use as a photoelastic coating as LCEs 1 and 2 require a washing stage following polymerization. As mentioned above, it seems plausible that the photoelastic response of LCE 3 could be increased to match, if not exceed, that of LCE 1 by the introduction of highly polar groups into the mesogenic structures. Additionally, we anticipate that materials of even greater performance (higher compliances and ductilities) could readily be created via alternative photopolymerizable chemistries such as thiol-acrylates, thiol-enes, and thiol-ynes. Another exciting prospect would be the use isotropic LCEs as surrogate materials for photoelastic studies of biological tissues. ${ }^{11}$ Such devices could be created by thermally initiated polymerization of LCE devices cast in molds.

In some applications of photoelastic materials, the cyclical deformation behavior of a device is of interest. In these cases, the photoelastic coatings would need to sustain cyclical straining without failure and they would need to provide consistent photoelastic responses over many cycles of testing. The cyclical mechanical testing performed here provide preliminary results demonstrating the promise of isotropic LCEs in cyclical photoelastic tests. While LCE 1 failed at 19 cycles of testing, LCE 3 last 88 cycles while LCE 2 passed the full 100 cycles of testing without failure. LCE 2 also demonstrated consistent a consistent peak stress of $1 \mathrm{MPa}$ throughout mechanical testing. Although LCE 3-the simplest of our LCEs to synthesize and most applicable to reflection photoelasticity-demonstrated strain hardening during the first few cycles and a gradual decline in the peak stress from cycles 20 to 88 , we believe that together our results indicate that future work could identify materials with facile synthesis 
and consistent photoelastic and mechanical behavior. We note that in many applications, devices would undergo cyclical strains of much smaller magnitude and as such our materials would be expected to undergo many more cycles of testing before failure. Future studies investigating suitable isotropic LCEs for cyclical photoelastic studies should employ quantitative measurements of the photoelastic response during cyclical testing.

Templated Isotropic Symmetry. Here, the process by which we synthesized LCE 1 -polymerization of a nematic precursor in the isotropic phase-yielded an isotropic elastomer. We see this both in the material's zero-order parameter when unstrained (Figure 4c) and its tensile mechanical behavior which is well fitted by eq 3 . This is in stark contrast to all other known LCE systems which, when processed in this manner, instead yield isotropic polydomain nematic elastomers (IPNEs). ${ }^{24,26,33,44,53}$ When below $T_{\mathrm{NI}}$, IPNEs are characterized by their opacity (due to the scattering of light by the misalignment of microscopic birefringent nematic domains) and by their "soft elastic" plateau in their tensile load curves (a result of the rotation of the domains to align with the applied stress axis). The subtle difference between LCE 1 and other LCEs is highly significant as it means LCE 1 can be templated by the symmetry of its polymerization state. This was something first proposed by P. G. de Gennes in 1969 (as a method to create a LCE) but deemed impossible for conventional, nonmesogenic elastomers as they relax out of their templated symmetry when the templating LC phase is washed from the material following polymerization. ${ }^{54}$ LCE 1 thus could be used to study the effect of chemically identical elastomers, templated with a variety of LC symmetries.

Strain-Induced Nematic Order. While it is well-known that nematic LCEs will undergo a softening of the nematic-toisotropic transition when under strain (so-called paranematic transitions), ${ }^{29}$ the observation of strain-induced nematic order parameters in completely isotropic elastomers has only recently been observed. $^{26-28}$ Here, LCEs 1, 2, and 3 all demonstrate linear increases in their nematic order parameter with strainin agreement with the prediction (eq 10) made using Warner and Terentjev theory. LCEs 1 and 3 have larger couplings, $a$, between the polymer chain and mesogenic groups $(0.51 \pm$ 0.02 and $0.66 \pm 0.02$, respectively), than LCE 2 (0.37 \pm 0.02 ) - a fact rationalized by the fact that LCEs 1 and 3 have mesogenic cross-linking groups which are main-chain in nature and so align strongly with the applied stress axis. The sidechain mesogenic groups in LCEs 1 and 3 therefore experience additional alignment effects from the mesogenic cross-linkers. Additionally, $a$ being greater for LCE 3 than for LCE 1 is likely to be a result of the larger mesogenic cores of M1 and M2 (used in LCE 3) compared to that of A6OCB (used in LCE 2).

The magnitude of the coupling term, $a$, observed in LCEs 1 and 3 is greater than that observed by Donovan et al. (maximum value of 0.36 at low strain). This result is surprising as the LCE prepared by Donovan et al. is a main-chain LCEthus one should expect a value of $a=1$ (eq 10). ${ }^{26}$ By comparison, one would expect the predominantly side-on, side-chain systems studied here to have a far lower value of $a$ given the mesogenic moieties are not directly bonded into the polymer backbone. Furthermore, Urayama et al. studied the strain-induced order parameter of a side-chain acrylate LCE at $414 \mathrm{~K}=140{ }^{\circ} \mathrm{C}\left(1.14 \times T_{\mathrm{NI}}\right)$ and found the order parameter to remain essentially at zero at all strains (maximum strain of 0.5 ). Again, this result is surprising as the material studied by Urayama et al. had a similar chemistry and synthesis route to LCE 2 (postwashing, the Urayama LCE was approximately $82 \% \mathrm{~A} 6 \mathrm{OCB}$ and $18 \%$ HDDA while LCE 2 is approximately $43 \% \mathrm{~A} 6 \mathrm{OCB}, 41 \% \mathrm{EHA}$, and $16 \% \mathrm{HDDA})$. It is not clear why the backbone-mesogen coupling of our LCEs are that much greater than that of the Urayama material. While tests performed on LCE 3 suggest our materials show little temperature dependence in a near ambient temperatures (Figure $4 \mathrm{~d}$ ), we may find $a$ decreases if tests were performed at much higher temperatures such as those used by Urayama et al.

In the Finkelmann materials, the strain-induced order parameters are highly sensitive to small changes in temperature. For example, Kaufhold et al. ${ }^{29}$ studied a polyacrylate LCE heated into the isotropic phase $\left(T_{\mathrm{NI}}=59^{\circ} \mathrm{C}\right)$. Under a bias-stress of $37 \mathrm{kPa}$, their LCE had an order parameter of 0.40 at $62{ }^{\circ} \mathrm{C}$ which decreased to 0.08 at $69{ }^{\circ} \mathrm{C}$. The authors concluded that applied bias stresses had the effect of shifting the $T_{\mathrm{NI}}$ of their material. By comparison, our measurements of LCE 3's dye order parameter at 10 and $22{ }^{\circ} \mathrm{C}$ showed no temperature dependence (values of $0.71 \pm 0.02$ and $0.74 \pm$ 0.05 measured, respectively, Figure $4 \mathrm{~d}$ ).

Lastly, the magnitude of the maximum order parameters observed in this work $(0.22 \pm 0.02$ and $0.11 \pm 0.02$ for LCEs 1 and 2, respectively, from PRS and $0.28 \pm 0.02$ for LCE 3 from the guest-host effect at $10{ }^{\circ} \mathrm{C}$ ) are values typical of the paranematic phase; the nematic liquid crystal phase usually exhibits order parameters of $\sim 0.4-0.6$. If the ductility of the materials was greater, then the results from Kaufhold et al. and Donovan et al. suggest that the order parameter may increase enough for the material to enter a biphasic regime of paranematic and nematic ordering. ${ }^{26,29}$

\section{CONCLUSIONS}

In this work, we describe three liquid crystal-based elastomeric materials which uniquely combine high strain optic coefficients, high compliances (both orders of magnitude greater than conventional materials), and easy synthesis routes. As such, our materials have several performance advantages over the existing commercial materials used for reflection photoelastic studies-particularly for the study of soft materials and devices. Our materials also have several advantages over previously described LCE-based strain sensors given their unique combination of a facile polymerization route, no requirement for liquid crystal alignment, minimal temperature dependence at ambient temperatures, and conventional isotropic elastomer mechanical behaviors. We expect that our LCE materials, in particular LCE 3 which has the simplest synthetic route, would be particularly suited to determining the stress distributions across biological tissues, soft biomedical implants, and soft robotics applications where new materials are required. ${ }^{11}$

The materials described contain liquid crystalline monomers and cross-linker groups but, unlike most materials formed from similar monomers, are polymerized in an isotropic phase and are thus templated with isotropic symmetry on a molecular level. Nematic ordering is only present when bias stresses are applied. Of note is the fact that LCE 1 , a chemistry we have studied extensively as a monodomain nematic LCE, is unlike conventional LCEs as it does not form a polydomain nematic LCE when polymerized in the isotropic phase but instead 
forms an isotropic elastomeric material. This ability to template the isotropic and nematic phases in this material opens the possibility of templating an elastomer with a wide variety of molecular orders.

\section{ASSOCIATED CONTENT}

\section{SI Supporting Information}

Supporting Information provided in the manuscript has been provided. A . The Supporting Information is available free of charge at https://pubs.acs.org/doi/10.1021/acs.macromol.9b02456.

Video of the LCE 1 sample showing the repeatability of the photoelastic response (AVI)

Video of the LCE 2 sample showing the repeatability of the photoelastic response (AVI)

Video of the LCE 3 sample showing the repeatability of the photoelastic response (AVI)

Data set relevant to the theory (PDF)

\section{AUTHOR INFORMATION}

\section{Corresponding Author}

Devesh Mistry - School of Physics and Astronomy, University of Leeds, Leeds LS2 9JT, United Kingdom; (1) orcid.org/0000-

0003-0012-6781; Email: deveshmistry@outlook.com

\section{Authors}

Maryam Nikkhou - School of Physics and Astronomy, University of Leeds, Leeds LS2 9JT, United Kingdom

Thomas Raistrick - School of Physics and Astronomy, University of Leeds, Leeds LS2 9JT, United Kingdom;

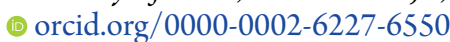

Mariam Hussain - School of Physics and Astronomy, University of Leeds, Leeds LS2 9JT, United Kingdom

Ethan I. L. Jull - School of Physics and Astronomy, University of Leeds, Leeds LS2 9JT, United Kingdom

Daniel L. Baker - School of Physics and Astronomy, University

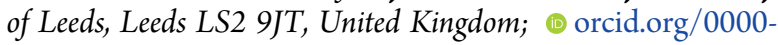
0002-5145-3320

Helen F. Gleeson - School of Physics and Astronomy, University of Leeds, Leeds LS2 9JT, United Kingdom

Complete contact information is available at: https://pubs.acs.org/10.1021/acs.macromol.9b02456

\section{Notes}

The authors declare no competing financial interest. Dataset for this paper can be found at the University of Leeds repository, at https://doi.org/10.5518/805.

\section{ACKNOWLEDGMENTS}

D.M. thanks UltraVision CLPL and the EPSRC for a CASE $\mathrm{PhD}$ studentship (award number 1611009), the Royal Commission for the Exhibition of 1851 for an Industrial Fellowship and the English Speaking Union for a Lindemann Trust Fellowship. E.I.L.J. acknowledges support from EPSRC in the form of a PhD studentship (EP/N509681/1). T.R. acknowledges funding from the Leeds University Alumni Fund. M.H. acknowledges funding from the Northern Triangle Initiative.

\section{REFERENCES}

(1) Zeng, H.; Wasylczyk, P.; Parmeggiani, C.; Martella, D.; Burresi, M.; Wiersma, D. S. Light-Fueled Microscopic Walkers. Adv. Mater. 2015, 27 (26), 3883-3887.

(2) Boothby, J. M.; Kim, H.; Ware, T. H. Shape Changes in Chemoresponsive Liquid Crystal Elastomers. Sens. Actuators, B 2017, 240, 511-518.

(3) Ware, T. H.; Biggins, J. S.; Shick, A. F.; Warner, M.; White, T. J. Localized Soft Elasticity in Liquid Crystal Elastomers. Nat. Commun. 2016, 7, 10781.

(4) Schuhladen, S.; Preller, F.; Rix, R.; Petsch, S.; Zentel, R.; Zappe, $\mathrm{H}$. Iris-Like Tunable Aperture Employing Liquid-Crystal Elastomers. Adv. Mater. 2014, 26 (42), 7247-7251.

(5) Mistry, D.; Connell, S. D.; Mickthwaite, S. L.; Morgan, P. B.; Clamp, J. H.; Gleeson, H. F. Coincident Molecular Auxeticity and Negative Order Parameter in a Liquid Crystal Elastomer. Nat. Commun. 2018, 9 (1), 5095.

(6) Giallorenzi, T.; Bucaro, J.; Dandridge, A.; Sigel, G.; Cole, J.; Rashleigh, S.; Priest, R. Optical Fiber Sensor Technology. IEEE J. Quantum Electron. 1982, 18 (4), 626-665.

(7) Wiśniewski, Z.; Wiśniewski, R.; Nowiński, J. L. Application of Foil Strain Gauges in High Pressure Research. Rev. Sci. Instrum. 2001, 72 (6), 2829-2831.

(8) Asundi, A.; Kishen, A. A Strain Gauge and Photoelastic Analysis of in Vivo Strain and in Vitro Stress Distribution in Human Dental Supporting Structures. Arch. Oral Biol. 2000, 45 (7), 543-550.

(9) Doyle, B. J.; Killion, J.; Callanan, A. Use of the Photoelastic Method and Finite Element Analysis in the Assessment of Wall Strain in Abdominal Aortic Aneurysm Models. J. Biomech. 2012, 45 (10), $1759-1768$.

(10) Strait, D. S.; Wang, Q.; Dechow, P. C.; Ross, C. F.; Richmond, B. G.; Spencer, M. A.; Patel, B. A. Modeling Elastic Properties in Finite-Element Analysis: How Much Precision Is Needed to Produce an Accurate Model? Anat. Rec., Part A 2005, 283A (2), 275-287.

(11) Tomlinson, R. A.; Taylor, Z. A. Photoelastic Materials and Methods for Tissue Biomechanics Applications. Opt. Eng. 2015, 54 (8), 081208.

(12) Sung, P.-C.; Wang, W.-C.; Hwang, C.-H.; Lai, G.-T. A LowLevel Stress Measurement Method by Integrating White Light Photoelasticity and Spectrometry. Opt. Laser Technol. 2018, 98, 3345 .

(13) Ozkir, S.; Terzioglu, H. Macro Design Effects on Stress Distribution around Implants: A Photoelastic Stress Analysis. Indian J. Dent. Res. 2012, 23 (5), 603.

(14) Bigoni, D.; Noselli, G. Localized Stress Percolation through Dry Masonry Walls. Part II - Modelling. Eur. J. Mech. A/Solids 2010, 29 (3), 299-307.

(15) Colla, C.; Gabrielli, E. Photoelasticity and DIC as Optical Techniques for Monitoring Masonry Specimens under Mechanical Loads. J. Phys.: Conf. Ser. 2017, 778 (1), 012003.

(16) Fernandes, C. P.; Glantz, P. O. J.; Svensson, S. A.; Bergmark, A. Reflection Photoelasticity: A New Method for Studies of Clinical Mechanics in Prosthetic Dentistry. Dent. Mater. 2003, 19 (2), 106117.

(17) Hirokawa, S.; Yamamoto, K.; Kawada, T. A Photoelastic Study of Ligament Strain. IEEE Trans. Rehabil. Eng. 1998, 6 (3), 300-308.

(18) Kawada, T.; Abe, T.; Yamamoto, K.; Hirokawa, S.; Soejima, T.; Tanaka, N.; Inoue, A. Analysis of Strain Distribution in the Medial Collateral Ligament Using a Photoelastic Coating Method. Med. Eng. Phys. 1999, 21 (5), 279-291.

(19) Fakhouri, S. F.; Novais-Shimano, S. G.; Shimano, M. M.; Defino, H.; Araujo, C. A.; Shimano, A. C. Photoelastic and Finite Element Stress Analysis of the Gap between the L4 and L5 Vertebrae. ISRN Mech. Eng. 2011, 2011, 1-6.

(20) Volpe, R. H.; Mistry, D.; Patel, V. V.; Patel, R. R.; Yakacki, C. M. Dynamically Crystalizing Liquid-Crystal Elastomers for an Expandable Endplate-Conforming Interbody Fusion Cage. $A d v$. Healthcare Mater. 2020, 9, 1901136. 
(21) Redner, A. S. Photoelastic Coatings. Exp. Mech. 1980, 20 (11), 403-408.

(22) Picot, O. T.; Dai, M.; Billoti, E.; Broer, D. J.; Peijs, T.; Bastiaansen, C. W. M. A Real Time Optical Strain Sensor Based on a Cholesteric Liquid Crystal Network. RSC Adv. 2013, 3 (41), 18794.

(23) Gleim, W.; Finkelmann, H. Thermoelastic and Photoelastic Properties of Crosslinked Liquid-Crystalline Side Chain Polymers. Makromol. Chem. 1987, 188 (6), 1489-1500.

(24) Schätzle, J.; Kaufhold, W.; Finkelmann, H. Nematic Elastomers: The Influence of External Mechanical Stress on the Liquid-Crystalline Phase Behavior. Makromol. Chem. 1989, 190 (12), 3269-3284.

(25) Lebar, A.; Cordoyiannis, G.; Kutnjak, Z.; Zalar, B. The Isotropic-to-Nematic Conversion in Liquid Crystalline Elastomers. In Liquid Crystal Elastomers: Materials and Applications; de Jeu, W. H., Ed.; Springer: Berlin/Heidelberg, 2010; pp 147-185 DOI: 10.1007/ 12_2010_103.

(26) Donovan, B. R.; Fowler, H. E.; Matavulj, V. M.; White, T. J. Mechanotropic Elastomers. Angew. Chem. 2019, 131 (39), 1388213886.

(27) Mistry, D. Synthetic Lenses for the Ageing Eye: A Motivation for Developing the Mechanical Applications of Liquid Crystal Elastomers, Thesis, University of Leeds, 2018.

(28) Mistry, D.; Gleeson, H. F. Mechanical Deformations and Applications of Liquid Crystal Elastomers in Novel Geometries. Presentation at the 2017 European Liquid Crystals Conference, 2017, Moscow.

(29) Kaufhold, W.; Finkelmann, H.; Brand, H. R. Nematic Elastomers, 1. Effect of the Spacer Length on the Mechanical Coupling between Network Anisotropy and Nematic Order. Makromol. Chem. 1991, 192 (11), 2555-2579.

(30) Mistry, D.; Morgan, P. B.; Clamp, J. H.; Gleeson, H. F. New Insights into the Nature of Semi-Soft Elasticity and "MechanicalFréedericksz Transitions" in Liquid Crystal Elastomers. Soft Matter 2018, 14 (8), 1301-1310.

(31) Lu, B. S.; Ye, F.; Xing, X.; Goldbart, P. M. Phenomenological Theory of Isotropic-Genesis Nematic Elastomers. Phys. Rev. Lett. 2012, 108 (25), 1-5.

(32) Fridrikh, S.; Terentjev, E. Polydomain-Monodomain Transition in Nematic Elastomers. Phys. Rev. E: Stat. Phys., Plasmas, Fluids, Relat. Interdiscip. Top. 1999, 60 (2), 1847-1857.

(33) Traugutt, N. A.; Volpe, R. H.; Bollinger, M. S.; Saed, M. O.; Torbati, A. H.; Yu, K.; Dadivanyan, N.; Yakacki, C. M. Liquid-Crystal Order during Synthesis Affects Main-Chain Liquid-Crystal Elastomer Behavior. Soft Matter 2017, 13 (39), 7013-7025.

(34) Haloi, D. J.; Roy, S.; Singha, N. K. Copper Catalyzed Atom Transfer Radical Copolymerization of Glycidyl Methacrylate and 2Ethylhexyl Acrylate. J. Polym. Sci., Part A: Polym. Chem. 2009, 47 (23), 6526-6533.

(35) Cowling, S. J.; Ellis, C.; Goodby, J. W. Anthraquinone Liquid Crystal Dichroic Dyes-a New Form of Chromonic Dye? Liq. Cryst. 2011, 38 (11-12), 1683-1698.

(36) Mistry, D.; Gleeson, H. F. Mechanical Deformations of a Liquid Crystal Elastomer at Director Angles between $0^{\circ}$ and $90^{\circ}$ : Deducing an Empirical Model Encompassing Anisotropic Nonlinearity. J. Polym. Sci., Part B: Polym. Phys. 2019, 57 (20), 13671377.

(37) Sims, M. T.; Abbott, L. C.; Cowling, S. J.; Goodby, J. W.; Moore, J. N. Molecular Design Parameters of Anthraquinone Dyes for Guest-Host Liquid-Crystal Applications: Experimental and Computational Studies of Spectroscopy, Structure, and Stability. J. Phys. Chem. C 2016, 120 (20), 11151-11162.

(38) Heilmeier, G. H.; Zanoni, L. A. GUEST-HOST INTERACTIONS in NEMATIC LIQUID CRYSTALS. a NEW ELECTROOPTIC EFFECT. Appl. Phys. Lett. 1968, 13 (3), 91-92.

(39) Heilmeier, G. H.; Castellano, J. A.; Zanoni, L. A. Guest-Host Interactions in Nematic Liquid Crystals. Mol. Cryst. 1969, 8 (1), 293304.

(40) Uchida, T.; Wada, M. Guest-Host Type Liquid Crystal Displays. Mol. Cryst. Liq. Cryst. 1981, 63 (1-4), 19-43.
(41) Zhang, Z.; Panov, V. P.; Nagaraj, M.; Mandle, R. J.; Goodby, J. W.; Luckhurst, G. R.; Jones, J. C.; Gleeson, H. F. Raman Scattering Studies of Order Parameters in Liquid Crystalline Dimers Exhibiting the Nematic and Twist-Bend Nematic Phases. J. Mater. Chem. C 2015, 3 (38), 10007-10016.

(42) Zhang, Z.; Kaur, S.; Kundu, B.; Sadashiva, B. K.; Gleeson, H. F. Observing the Emergence of Phase Biaxiality in a Polar Smectic A System via Polarised Raman Spectroscopy. J. Mater. Chem. C 2017, 5 (5), 1195-1205.

(43) Southern, C. D.; Gleeson, H. F. Using the Full Raman Depolarisation in the Determination of the Order Parameters in Liquid Crystal Systems. Eur. Phys. J. E: Soft Matter Biol. Phys. 2007, 24 (2), 119-127.

(44) Warner, M.; Terentjev, E. M. Liquid Crystal Elastomers; Clarendon Press: Oxford, 2013 DOI: 10.1017/ CBO9781107415324.004.

(45) Higaki, H.; Takigawa, T.; Urayama, K. Nonuniform and Uniform Deformations of Stretched Nematic Elastomers. Macromolecules 2013, 46 (13), 5223-5231.

(46) Finkelmann, H.; Greve, A.; Warner, M. The Elastic Anisotropy of Nematic Elastomers. Eur. Phys. J. E: Soft Matter Biol. Phys. 2001, 5 (3), 281-293.

(47) Gent, A. N. Stress-Induced Birefringence of Swollen Polymer Networks. Macromolecules 1969, 2 (3), 262-265.

(48) Muller, R.; Pesce, J. J. Stress-Optical Behaviour near the Tgand Melt Flow-Induced Anisotropy in Amorphous Polymers. Polymer 1994, 35 (4), 734-739.

(49) Arruda, E. M.; Boyce, M. C. Evolution of Plastic Anisotropy in Amorphous Polymers during Finite Straining. Int. J. Plast. 1993, 9 (6), 697-720.

(50) Roland, C. M. Viscoelastic Behavior of Rubbery Materials, $1^{\text {st }}$ ed.; Oxford University Press: Oxford, 2011 DOI: 10.1093/acprof:oso/ 9780199571574.001.0001.

(51) Prussin, S.; Stevenson, A. Strain-Optic Coefficient of Silicon for Infrared Light [15]. J. Appl. Phys. 1959, 30 (3), 452-453.

(52) Simha, N. K.; Jin, H.; Hall, M. L.; Chiravarambath, S.; Lewis, J. L. Effect of Indenter Size on Elastic Modulus of Cartilage Measured by Indentation. J. Biomech. Eng. 2007, 129 (5), 767.

(53) Urayama, K.; Kohmon, E.; Kojima, M.; Takigawa, T. Polydomain-Monodomain Transition of Randomly Disordered Nematic Elastomers with Different Cross-Linking Histories. Macromolecules 2009, 42 (12), 4084-4089.

(54) De Gennes, P. G. Possibilites Offertes Par La Reticulation de Polymeres En Presence d'un Cristal Liquide. Phys. Lett. A 1969, 28 (11), 725-726. 